Pacific Journal of Mathematics

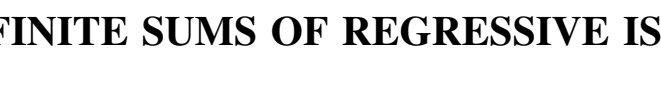




\title{
ON FINITE SUMS OF REGRESSIVE ISOLS
}

\author{
JOSEPH BARBACK
}

\begin{abstract}
Our paper deals with isols that can be represented as a finite sum of regressive isols, and with recursive functions of one variable that have canonical extensions that map isols such as these into the isols.
\end{abstract}

1. Preliminaries. We shall use familiar notation in the theory of isols. We denote by $\omega, \Lambda$ and $\Lambda_{R}$ respectively, the sets of nonnegative integers (numbers), isols, and regressive isols. If $f$ is any recursive function, in any number of variables, then $f_{i}$ denotes the canonical extension of $f$ to the isols. The degree of unsolvability of a regressive isol is defined in the following way, as it is introduced in [3]. Each regressive isol contains a retraceable set, and all retraceable sets that belong to the same regressive isol will have the same Turing degree of unsolvability. If $a$ is a regressive isol, then its degree of unsolvability is defined to be the Turing degree of any retraceable set that is a member of $a$. For a regressive isol $a, \Delta_{a}$ will denote the degree of unsolvability of $a$. It is proved in [3], that if $a$ and $b$ are any infinite regressive isols, then

$$
\begin{array}{ll}
\text { 1. } & a \leqq b \longrightarrow \Delta_{a}=\Delta_{b}, \\
\text { 2. } & a \leqq * b \longrightarrow \Delta_{b} \leqq \Delta_{a}, \text { and } \\
\text { 3. } & a+b \text { regressive implies } \Delta_{a}=\Delta_{b} .
\end{array}
$$

Several times in the paper we use refinement property of isols. This property refers to the following feature of the isols, and it is obtained in [6, corollary to Theorem 19]. If $y$ and $a_{0}, \cdots, a_{m}$ are any isols, and

$$
y \leqq a_{0}+\cdots+a_{m},
$$

then there will be isols $y_{0}, \cdots, y_{m}$ with $y=y_{0}+\cdots+y_{m}$ and with each $y_{i} \leqq a_{i}$.

2. Finite sums of regressive isols. Let $m \geqq 1$ be any number. We set

$$
m \Lambda_{R}=\left\{a_{0}+\cdots+a_{m-1} \mid a_{0}, \cdots, a_{m-1} \in \Lambda_{R}\right\} .
$$

When $m=1$ then $m \Lambda_{R}$ is simply the collection of all regressive isols. It is easy to see from their definitions, that one has

$$
\omega \subset \Lambda_{R} \subset 2 \Lambda_{R} \subset 3 \Lambda_{R} \subset \cdots .
$$


Each of the inclusions that appear above will be proper. That the first two are, follows from the work of J. C. E. Dekker in [3]. Each of the remaining ones may be shown to proper by an approach similar to the one applied in [3], for showing the second inclusion is proper. We do this next. Let $a_{0}, \cdots, a_{m}$ be $m+1$ infinite regressive isols, selected to have mutually distinct degrees of unsolvability. Then, it is clear, the isol $y=a_{0}+\cdots+a_{m}$ is in $(m+1) \Lambda_{R}$. But $y$ is not a member of $m \Lambda_{R}$. For if otherwise, it then would follow, from the refinement property for isols, that there exist infinite $b_{i}$ and $b_{j}$ with $i \neq j, b_{i} \leqq a_{i}, b_{j} \leqq a_{j}$, and $b_{i}+b_{j}$ regressive. This would mean that $b_{i}$ and $b_{j}$ have the same degree of unsolvability, and therefore also, that $\alpha_{i}$ and $\alpha_{j}$ have the same degree of unsolvability, and thus contradicting our selection of the isols $a_{i}$ and $a_{j}$. Thus each $m \Lambda_{R}$ is a proper subset of $(m+1) \Lambda_{R}$. It follows from this fact that also each of the collections $m \Lambda_{R}$ for $m \geqq 1$ is not closed under addition.

Let $m \geqq 1$ and let $y \in m \Lambda_{R}$. Then it is easy to see that each of the isols $y+1, y+2, \cdots$ and $2 y, 3 y, \cdots$ is also a member of $m A_{R}$. These are similar features that the collections $m \Lambda_{R}$ share with the collection of regressive isols. In contrast, it will not necessarily be true that $y^{2}$ is a member of $m \Lambda_{I}$. This fact will follow from Proposition 2 that appears below. The next result establishes two analogues for the collections $m \Lambda_{R}$ of properties well-known for the regressive isols.

Proposition 1. Let $m \geqq 1$ and let $y \in m \Lambda_{r}$. Then for all isols $a$ and $b$,

$$
\begin{aligned}
& a \leqq y \Rightarrow a \in m \Lambda_{R}, \text { and } \\
& (a \leqq y \text { and } b \leqq y) \Longrightarrow a+b \in m \Lambda_{R} .
\end{aligned}
$$

Proof. In the special case $m=1$ then both results are proved by Dekker in [3]. For the general setting, let $y=y_{0}+\cdots+y_{m-1}$ with each $y_{i}$ a regressive isol. If $a \leqq y$, then by the refinement property, it will follow that $a=a_{0}+\cdots+a_{m-1}$ where for each $i$, one has $a_{i} \leqq y_{i}$. Then each of the isols $a_{i}$ is regressive, and therefore $a \in m \Lambda_{R}$. This verifies (1). To verify statement (2), assume both $a \leqq y$ and $b \leqq y$. Then we obtain,

$$
\begin{aligned}
& a=a_{0}+\cdots+a_{m-1}, \quad \text { and } \\
& b=b_{0}+\cdots+b_{m-1},
\end{aligned}
$$

with $a_{i} \leqq y_{2}$ and $b_{\imath} \leqq y_{\imath}$ for each $i$. Since each $y_{i}$ is regressive, then also is each of the sums $a_{i}+b_{i}$. Hence the isol 


$$
a+b=\left(a_{0}+b_{0}\right)+\cdots+\left(a_{m-1}+b_{m-1}\right)
$$

belongs to $m \Lambda_{R}$. This verifies (2), and completes the proof.

In the next result it is shown that when $a$ and $b$ are each infinite regressive isols and $y=a+b$, then the degrees $\Delta_{a}$ and $\Delta_{b}$ are comparable if $y^{2}$ is an element of $2 \Lambda_{R}$. If we would therefore select such isols $a$ and $b$ to have incomparable degrees, then the associated isol $y$ will belong to $2 \Lambda_{R}$ but the isol $y^{2}$ will not. Our proof below will assume some familiarity with the notion of the minimum of two regressive isols and with the relation of $\leqq *$ among isols. Each of these notions is introduced and studied in [3].

Proposition 2. Let $a$ and $b$ be infinite regressive isols, and let $y=a+b$. Let $u$ and $v$ be regressive isols with $y^{2}=u+v$. Then either $\Delta_{a} \leqq \Delta_{b}$ or $\Delta_{b} \leqq \Delta_{a}$.

Proof. Because $y=a+b$ it follows that

$$
a^{2}+2 a b+b^{2}=u+v,
$$

and therefore also, since $\min (a, b) \leqq a b$, one has

$$
a+\min (a, b)+b \leqq u+v .
$$

It is known, from [3], that $\min (a, b) \leqq{ }^{*} a$ and $\min (a, b) \leqq{ }^{*} b$. From the refinement property of isols, it follows from (1) that one of the cases below will necessarily occur.

Case 1. There are infinite regressive isols $a^{\prime}$ and $z$ with $a^{\prime} \leqq a$, $z \leqq \min (a, b)$, and either $a^{\prime}+z \leqq u$ or $a^{\prime}+z \leqq v$.

In this case then $a^{\prime}$ and $z$ will have the same degree of unsolvability. The degree of $a^{\prime}$ is the same as the degree of $a$. The degree of $z$ will be the same as the degree of $\min (a, b)$, which is known to have the property $\Delta_{b} \leqq \Delta_{\min }(a, b)$. Thus it will follow that $\Delta_{b} \leqq \Delta_{a}$.

Case 2. There are infinite regressive isols $b^{\prime}$ and $z$ with $b^{\prime} \leqq b$, $z \leqq \min (a, b)$, and either $b^{\prime}+z \leqq u$ or $b^{\prime}+z \leqq v$.

In this case, it will follow, in a similar way as in Case 1, that $\Delta_{a} \leqq \Delta_{b}$. We will omit the details for showing this.

Case 3. There are infinite regressive isols $a^{\prime}$ and $b^{\prime}$ with $a^{\prime} \leqq a$, $b^{\prime} \leqq b$, and either $a^{\prime}+b^{\prime} \leqq u$ or $a^{\prime}+b^{\prime} \leqq v$.

In this case, it will follow that both $a^{\prime}$ and $b^{\prime}$ have the same degree of unsolvability. Since both $a^{\prime}$ and $a$, and $b^{\prime}$ and $b$, share the same degree one obtains $\Delta_{a}=\Delta_{b}$. 
In view of the three cases considered above, it follows that the degrees $\Delta_{a}$ and $\Delta_{b}$ will be comparable, and this completes the proof.

3. Canonical extensions of recursive functions. Let $m \geqq 1$. In this section we characterize the recursive functions of one variable with the property that their canonical extensions map $m \Lambda_{R}$ into $\Lambda$. We are grateful to E. Ellentuck for showing us how to obtain this result based upon his work in [7], and our proof of Theorem 2 is based upon his ideas. We also obtain in this section a characterization of the recursive functions of one variable with canonical extensions that map $m \Lambda_{R}$ into the isols and that are order preserving on $m \Lambda_{R}$ with respect to the relation of $\leqq$ among isols. In the case $m=1$ the latter result was first proved by F. Sansone in [11]. We first introduce some definitions and results, taken mainly from the work of Ellentuck in [7].

Let $n \geqq 1$. For $x \in \omega^{n}$, we write $x=\left(x_{0}, \cdots, x_{n-1}\right)$, with $x_{i}$ denoting the $(i+1)$ st component of $x$, and set $\min x=\operatorname{minimum}\left(x_{0}, \cdots, x_{n-1}\right)$. For $k \in \omega$, we write $k$ for the particular element of $\omega^{n}$, each of whose components is equal to $k$. For all $x, y \in \omega^{n}$ (respectively $\Lambda^{n}$ ), we write $x+y$ for the element of $\omega^{n}$ (respectively $\left.\Lambda^{n}\right)$ with $(x+y)_{2}=$ $x_{i}+y_{i}$. Let $g$ be any function from $\omega^{n}$ into $\omega$. With $g$ associate a new function $\hat{g}$ from $\omega^{n}$ into $\omega$, defined by

$$
\begin{aligned}
& \hat{g}(x)=0 \quad \text { if } \quad \min x=0, \\
& \hat{g}(x)=g(y) \quad \text { where } y+1=x, \quad \text { if } \quad \min x \geqq 1 .
\end{aligned}
$$

For each $j<n$ define $n$-ary functions $E_{j} g$ and $\Delta_{j} g$ by

$$
\begin{aligned}
& E_{j} g(x)=g(y) \text { where } y_{j}=x_{j}+1 \text { and } y_{i}=x_{\imath} \text { for } i \neq j, \text { and } \\
& \Delta_{j} g(x)=\left(E_{j} g(x)\right)-g(x) .
\end{aligned}
$$

Let $\Delta=\Delta_{0} \cdots \Delta_{n-1}$ be the composition of the $\Delta_{j}^{\prime} \mathrm{s}$. A function $g: \omega^{n} \rightarrow \omega$ is called recursive increasing if $g$ is recursive and $\Delta \widehat{g}(x) \geqq 0$ for every $x \in \omega^{3}$, and is called eventually recursive increasing if there is a value $k \in \omega^{2}$ such that the function $g(x+k)$ is recursive increasing.

In the special case that we are considering a function of only one variable, as $f(x)$, then we let $x=x_{0}$ and $\Delta f(x)=\Delta_{0} f(x)$, and define, for each number $m$,

$$
\Delta^{0} f(x)=f(x) \text { and } \Delta^{m+1} f(x)=\Delta\left(\Delta^{m} f(x)\right) .
$$

Later in the paper we will want to examine when for a particular function of one variable, as $f(x)$, the associated function $\Delta^{m} f(x)$ is eventually recursive increasing. From the definition above, it is 
easy to check that in this case one has the following characterization: $\Delta^{m} f(x)$ is eventually recursive increasing if and only if $f(x)$ is recursive and there exists a number $k \in \omega$ such that $\Delta^{m+1} f(x+k) \geqq 0$ for all numbers $x$. It is this latter characterization that is applied later in the paper.

We introduce next the ideas associated with the concept of an almost recursive increasing function; as these are introduced in [7]. If $\alpha$ is a finite set then the cardinality of $\alpha$ is denoted by card. $\alpha$. Let $\nu(0)=\phi$ and for $n \geqq 1$, let $\nu(n)=(0, \cdots, n-1)$. Then card. $\nu(n)=$ $n$ for each number $n$. Let $h, \alpha, d$ and $j$ together satisfy the following properties:

$$
\begin{aligned}
& \alpha \subset \nu(n), h: \alpha \rightarrow \omega, \text { card. }(\nu(n)-\alpha)=d \text { with } d>0, \text { and } \\
& j: \nu(d) \rightarrow(\nu(n)-\alpha) \text { is strictly increasing. }
\end{aligned}
$$

With $h$ associate a new function $h_{n}^{*}: \omega^{d} \rightarrow \omega^{n}$, defined in the following way: $h_{n}^{*}(x)=y$ where $y_{i}=h(i)$ for $i \in \alpha$, and $y_{j(2)}=x_{i}$ for $i<d$.

A function $g: \omega^{n} \rightarrow \omega$ is called almost recursive increasing, if for every function $h$ that satisfies (1), the composition function $g \circ h_{n}^{*}$ is eventually recursive increasing. An almost recursive increasing function will be recursive, and also, will be eventually recursive increasing, for one may select as a function $h$ in (1) one with $\alpha=\phi$. The following theorem is proved in [7].

TheOREM 1. (Ellentuck). Let $n \geqq 1$ and let $g: \omega^{n} \rightarrow \omega$ be a recursive function. Then $g_{A}$ maps $\Lambda_{R}^{n}$ into $\Lambda$ if and only if $g$ is an almost recursive increasing function.

We characterize next the recursive functions of one variable with canonical extensions that map $m \Lambda_{R}$ into $\Lambda$. Our proof below is due to E. Ellentuck.

THEOREM 2. Let $m \geqq 1$ and let $f(x)$ be a recursive function. Then $f_{\Lambda}$ maps $m \Lambda_{R}$ into $\Lambda$ if and only if, for each $j<m, \Delta^{j} f(x)$ is an eventually recursive increasing function.

Proof. (Ellentuck). Let the function $g: \omega^{m} \rightarrow \omega$ be defined by $g\left(x_{0}, \cdots, x_{m-1}\right)=f\left(x_{0}+\cdots+x_{m-1}\right)$. Then $g$ is a recursive function. Also, $g\left(x_{0}, \cdots, x_{m-1}\right)=f\left(x_{0}+\cdots+x_{m-1}\right)$, for all isols $x_{0}, \cdots, x_{m-1}$. From Theorem 1, one obtains the following equivalent statements:

$f_{\Lambda}$ maps $m \Lambda_{R}$ into $\Lambda$, if and only if

$g_{\Lambda}$ map $\Lambda_{R}^{m}$ into $\Lambda$, if and only if

$g$ is almost recursive increasing . 
To obtain the desired result, we will verify that $g$ is almost recursive increasing, if and only if, for each $j<m, \Delta^{j} f(x)$ is an eventually recursive increasing function.

Let $j \geqq 1$. Consider the following set of equations.

$$
\begin{aligned}
\Delta \hat{g}\left(x_{0}\right. & \left.+1, \cdots, x_{j-1}+1,1, \cdots, 1\right) \\
& =\left(\Pi_{i<j} \Delta_{i}\right) \hat{g}\left(x_{0}+1, \cdots, x_{j-1}+1,1, \cdots, 1\right) \\
& =\left(\Pi_{i<j} \Delta_{i}\right) g\left(x_{0}, \cdots, x_{j-1}, 0, \cdots, 0\right) \\
& =\left(\Pi_{i<j} \Delta_{i}\right) f\left(x_{0}+\cdots+x_{j-1}\right) \\
& =\left(\Delta^{j} f\right)\left(x_{0}+\cdots+x_{j-1}\right) .
\end{aligned}
$$

The first three equalities follow from definitions. The last equality holds because $\Delta_{r} f\left(x_{0}+\cdots+x_{j-1}\right)=\Delta_{s} f\left(x_{0}+\cdots+x_{j-1}\right)$, for all $r, s<$ $j$. Therefore, the particular function $g\left(x_{0}, \cdots, x_{j-1}, 0, \cdots, 0\right)$ is recursive increasing, if and only if, $\Delta^{j-1} f(x)$ is recursive increasing. Combining the definition of an almost recursive increasing function and the way in which the function $g$ is defined in terms of $f$, it follows from the equations in (2) that $g$ is almost recursive increasing if and only if $\Delta^{j} f(x)$ is eventually recursive increasing for each $j<m$. This completes the proof.

Let $m \geqq 1$ and let $f(x)$ be a recursive function. We say that $f_{A}$ is order preserving on $m \Lambda_{R}$, if $f_{A}$ maps $m \Lambda_{R}$ into $\Lambda$, and if for all $a, b \in m \Lambda_{R}$ one has $a \leqq b$ implies $f_{\Lambda}(a) \leqq f_{\Lambda}(b)$. F. Sansone, in [11], characterized the recursive functions $f$ that have $f_{A}$ order preserving on $\Lambda_{R}$. In the next result we consider the corresponding problem for an arbitrary value for $m$.

THEOREM 3. Let $m \geqq 1$ and let $f(x)$ be a recursive function of one variable. Let $\Delta^{j} f(x)$ be eventually (recursive) increasing, for each $j<m$. Then the following three conditions are equivalent:

(1) $\Delta^{m} f(x)$ is eventually recursive increasing,

(2) $f_{\Lambda}\left(x_{0}+\cdots+x_{m-1}\right) \leqq f_{A}\left(x_{0}+\cdots+x_{m-1}+x_{m}\right)$, for all regressive isols $x_{0}, \cdots, x_{m}$,

(3) $f_{A}$ is order preserving on $m \Lambda_{R}$.

Proof. For the case $m=1$, the equivalence of statements (1) and (3) was proved by F. Sansone in [11].

Define the functions $g$ and $h$, from $\omega^{m+1}$ into $\omega$, by

$$
\begin{gathered}
g\left(x_{0}, \cdots, x_{m}\right)=f\left(x_{0}+\cdots+x_{m}\right), \\
h\left(x_{0}, \cdots, x_{m}\right)=g\left(x_{0}, \cdots, x_{m}\right)-g\left(x_{0}, \cdots, x_{m-1}, 0\right) .
\end{gathered}
$$


From these equations it follows that both $g$ and $h$ are recursive functions, and, in addition, one also obtains, for all isols $x_{0}, \cdots, x_{m}$,

$$
h_{A}\left(x_{0}, \cdots, x_{m}\right)=f_{A}\left(x_{0}+\cdots+x_{m}\right)-f_{A}\left(x_{0}+\cdots+x_{m-1}\right) .
$$

Let us assume that statement (1) holds. Let $x_{0}, \cdots, x_{m}$ be any regressive isols. By Theorem 2, both $f_{i}\left(x_{0}+\cdots+x_{m}\right)$ and $f_{A}\left(x_{0}+\cdots+x_{m-1}\right)$ are isols. Also, from (4), it follows that

$$
f_{A}\left(x_{0}+\cdots+x_{m-1}\right) \leqq f_{A}\left(x_{0}+\cdots+x_{m}\right)
$$

holds, if and only if, $h_{A}\left(x_{0}, \cdots, x_{m}\right)$ is an isol. Therefore, (2) will follow if $h_{A}$ maps $\Lambda_{R}^{m+1}$ into $\Lambda$. By Theorem 1 , this property is equivalent to $h$ being an almost recursive increasing function. We now verify that $h$ has this feature.

Let $d \leqq m+1$ and consider the following two separate computations that involve $\Delta \hat{h}$.

Case 1. When $d \leqq m$. Let $k \in \omega$ and let $u=x_{0}+\cdots+x_{d-1}$.

$$
\begin{aligned}
\Delta \hat{h}\left(x_{0}\right. & \left.+1, \cdots, x_{d-1}+1,1, \cdots, 1, k+1\right) \\
= & \Delta h\left(x_{0}, \cdots, x_{d-1}, 0, \cdots, 0, k\right) \\
= & \left(\Pi_{j<d} \Delta_{j}\right) h\left(x_{0}, \cdots, x_{d-1}, 0, \cdots, 0, k\right) \\
= & \left(\Pi_{j<d} \Delta_{j}\right)\left[g\left(x_{0}, \cdots, x_{d-1}, 0, \cdots, 0, k\right)\right. \\
& \left.-g\left(x_{0}, \cdots, x_{d-1}, 0, \cdots, 0,0\right)\right],
\end{aligned}
$$

and, by representing the last expression in terms of $u$ and the function $f$, the equations may be continued, to give,

$$
\begin{aligned}
& =\Delta^{d}[f(u+k)-f(u)] \\
& =\Delta^{d}\left[\Sigma_{i<k} \Delta f(u+i)\right] \\
& =\Sigma_{i<k} \Delta^{d+1} f(u+i) .
\end{aligned}
$$

Case 2. When $d=m+1$. Let $v=x_{0}+\cdots+x_{m}$.

$$
\begin{aligned}
\Delta \hat{h}\left(x_{0}\right. & \left.+1, \cdots, x_{m}+1\right)=\Delta h\left(x_{0}, \cdots, x_{m}\right) \\
& =\Delta\left[g\left(x_{0}, \cdots, x_{m}\right)-g\left(x_{0}, \cdots, x_{m-1}, 0\right)\right] \\
& =\left(\Pi_{j<m+1} \Delta_{j}\right)\left[g\left(x_{0}, \cdots, x_{m}\right)-g\left(x_{0}, \cdots, x_{m-1}, 0\right)\right] \\
& =\left(\Pi_{j<m+1} \Delta_{j}\right) g\left(x_{0}, \cdots, x_{m}\right) \\
& =\Delta^{m+1} f(v) .
\end{aligned}
$$

By combining both the hypothesis in the theorem and the assumed property in statement (1), it follows that each of the expressions computed for $\Delta \hat{h}$, in Cases 1 and 2 , is nonnegative. As in the proof of Theorem 2, this fact implies that $h$ is an almost increasing function. Thus we obtain statement (2). 
Let us assume now that statement (2) holds. Let $a$ and $b$ be any members of $m \Lambda_{R}$ with $a \leqq b$. Let

$$
b=b_{0}+\cdots+b_{m-1},
$$

with each of $b_{0}, \cdots, b_{m-1}$ a regressive isol. Because $a \leqq b$ it follows that we may represent $a$ as,

$$
a=a_{0}+\cdots+a_{m-1},
$$

where for each $i, a_{\imath}$ is regressive and $a_{i} \leqq b_{i}$. For each $i$ let the isol $r_{i}$ be defined by $r_{i}=b_{i}-a_{i}$. Let $r=r_{0}+\cdots+r_{m-1}$. Then each $r_{i}$ is regressive and $a+r=b$. We may now obtain the desired result, statement (3), by successively applying the property given in statement (2), as follows:

$$
\begin{aligned}
f_{A}(a) & =f_{A}\left(a_{0}+\cdots+a_{m-1}\right) \\
& \leqq f_{A}\left(a_{0}+\cdots+a_{m-1}+r_{0}\right) \\
& =f_{A}\left(b_{0}+a_{1}+\cdots+a_{m-1}\right) \\
& \vdots \\
& =f_{\Lambda}\left(b_{0}+\cdots+b_{m-1}\right) \\
& =f_{\Lambda}(b) .
\end{aligned}
$$

To complete the proof we now show that statement (3) implies statement (1). Assume statement (1) does not hold. Then, from Theorem 2, the canonical extension of the function $\Delta f(x)=f(x+1)$ $f(x)$ to the isols will not map $m \Lambda_{R}$ into $\Lambda$. There then will exist an isol $a \in m \Lambda_{R}$ with $f_{A}(a+1)-f(a)$ being a value in the isolic integers but not in the isols. This is equivalent to having, $f_{\Lambda}(a) \leqq f_{\Lambda}(a+1)$ not holding true. Since $a \leqq a+1$, and, $a \in m \Lambda_{R}$ implies $a+1 \in m \Lambda_{R}$, it follows that $f_{A}$ is not order preserving on $m \Lambda_{R}$. Hence, if statement (1) does not hold, then statement (2) does not hold also. This completes our proof.

EXAMPLE. We would like to illustrate some of the ideas of the previous discussion with one example. Let the function $f(x)$ be defined by $f(x)=\left[\begin{array}{c}x+1 \\ 2\end{array}\right] \cdot\left[\begin{array}{c}x+4 \\ 2\end{array}\right]$, where $\left[\begin{array}{l}y \\ 2\end{array}\right]$ denotes the greatest integer obtained when $y$ is divided by 2 . It is easy to to see that $f(x)$ is both a recursive and an increasing function. It is also easy to verify, perhaps easiest by computing the associated difference table, that the function $\Delta f$ is neither increasing nor eventually increasing. Hence, by Theorem $2, f_{A}$ does not map $2 \Lambda_{R}$ into $\Lambda$. On the other hand, the function $f^{2}(x)=(f(x))^{2}$ is recursive and increasing, and, in addition, has the feature that it's first difference function 
$\Delta f^{2}$ is also increasing. This fact can perhaps best be recognized by constructing a difference table for the function $f^{2}(x)$. Thus, while $f_{A}$ does not map $2 \Lambda_{R}$ into $\Lambda$, the function $f^{2}$, will. We can apply these properties to illustrate one feature in the nature of isols. Let $a$ and $b$ be regressive isols such that $f_{\Lambda}(a+b) \notin \Lambda$. If we set $y=$ $f_{\Lambda}(a+b)$ then we obtain an example of an isolic integer $y$, such that neither $y$ nor $-y$ is an isol, and $y^{2}$ is an isol. (This feature of the isols is well-known now, and we first learned about it from A. Nerode.)

REMARK. We would like to indicate some connections of our work in this section with combinatorial functions and their properties.

Let $m \geqq 1$ and let $f(x)$ be a recursive function. We say $f(x)$ is increasing of order $m$, if $\Delta^{0} f, \cdots, \Delta^{m-1} f$ are each increasing functions; and say it is eventually increasing of order $m$, if for some number $c$ the function $f(x+c)$ is increasing of order $m$. If, for each $m \in$ $\omega, f(x)$ is increasing of order $m$, then $f(x)$ is said to be a combinatorial function. This definition of a combinatorial function agrees with the familiar one introduced by J. Myhill in [9]. We would like to assume that the reader is familiar with some basic features of combinatorial functions. We note that $f(x)$ will be eventually increasing in each order of $m$, if there is a sequence of numbers $c_{0}, c_{1}, \cdots$ such that, for each $m$ the function $f\left(x+c_{m}\right)$ is increasing of order $m$. Also, $f(x)$ is called eventually combinatorial, if there is a constant $c$ such that $f(x+c)$ is combinatorial. Let

$$
\omega \Lambda_{R}=\bigcup_{\substack{m<\infty \\ m \neq 0}} m \Lambda_{R}
$$

Then, from Theorem 2, it follows that $f_{A}$ maps $m \Lambda_{R}$ into $\Lambda$ if and only if $f$ is eventually increasing of order $m$. Hence $f_{A}$ maps $\omega \Lambda_{R}$ into $A$ if and only if $f$ is eventually increasing in each order of $m$. From the work of A. Nerode, in [10], one has that $f_{4}$ maps $\Lambda$ into $\Lambda$ if and only if $f$ is eventually combinatorial.

If $f(x)$ is eventually combinatorial, then it is eventually increasing in each order of $m$; in this case the sequence of associated values of $c_{i}$ can be selected to be constant beyond some point. The converse is not true, as there exist recursive functions that are not eventually combinatorial, yet that are eventually increasing in each order of $m$. Such a function $g(x)$ may be constructed in the following manner; below is an array with some of the initial values and associated difference values for $g(x)$. Values along the diagonals, in the table following, for the function $g(x)$ and its differences will all be constant. 


$\begin{array}{rrrrrrr}x & g(x) & \Delta g(x) & \Delta^{2} g(x) & \Delta^{3} g(x) & \Delta^{4} g(x) & \Delta^{5} g(x) \\ 0 & 2 & -1 & 3 & -4 & 7 & -11 \\ 1 & 1 & 2 & -1 & 3 & -4 & 7 \\ 2 & 3 & 1 & 2 & -1 & 3 & -4 \\ 3 & 4 & 3 & 1 & 2 & -1 & 3 \\ 4 & 7 & 4 & 3 & 1 & 2 & -1 \\ 5 & 11 & 7 & 4 & 3 & 1 & 2\end{array}$

Recursive functions of this type will have canonical extensions which map $\omega \Lambda_{R}$ into $\Lambda$, but not map $\Lambda$ into $\Lambda$. It is easy to find isols a with $a \in \Lambda-\omega \Lambda_{R}$, for we may let $a$ be any infinite isol containing an infinite set which contains no infinite regressive subset.

Additional remark. In a recent paper, [8], V. L. Mikheev introduced some classes of functions like the ones we also studied in this paper.

\section{REFERENCES}

1. J. Barback, Recursive functions and regressive isols, Math. Scand., 15 (1964), 29-42. 2. - Two notes on recursive functions and regressive isols, Trans. Amer. Math. Soc., 144 (1969), 77-94.

3. J. C. E. Dekker, The minimum of two regressive isols, Math. Z., 83 (1964), 345-366.

4. - Infinite series of isols, Proc. Symposia Pure Math., 5 (1962), 77-96.

5. — Regressive isols, Sets, Models and Recursion Theory, North-Holland Publ., Amsterdam, 1967.

6. J. C. E Dekker and J. Myhill, Recursive equivalence types, Univ. California Publ. Math., (N. S.) 3 (1960), 67-213.

7. E. Ellentuck, On the form of functions which preserve regressive isols, Compositio Math., 26 (1973), 283-302.

8. V. L. Mikheev, A hierarchy of independent w-processions of cosimple isols, Algebra i Logika (Russian), 17 (1978), 56-78.

9. J. Myhill, Recursive equivalence types and combinatorial functions, Proc. of the International Congress in Logic and Methodology of Science, Stanford (1960), 46-55.

10. A. Nerode, Extensions to isols, Ann. of Math., 73 (1961), 362-403.

11. F. Sansone, On order-preserving extensions to regressive isols, Michigan Math., 13 (1966), 353-355.

Received June 6, 1980 and in revised form September 17, 1980.

Suny COLlege

BufFalo, NY 14222 


\section{PACIFIC JOURNAL OF MATHEMATICS}

\section{EDITORS}

DONALD BABBITT (Managing Editor)

University of California

Los Angeles, California 90024

Hugo RossI

University of Utah

Salt Lake City, UT 84112

C. C. MOORE and ANDREW OGG

University of California

Berkeley, CA 94720

\section{J. DugundJI}

Department of Mathematics University of Southern California Los Angeles, California 90007

R. Finn and J. Milgram Stanford University

Stanford, California 94305

\section{ASSOCIATE EDITORS}
R. ARENS
E. F. BeCKENBACH
B. H. Neumann
F. WoLF
K. YoSHIDA

\section{SUPPORTING INSTITUTIONS}

UNIVERSITY OF ARIZONA

UNIVERSITY OF BRITISH COLUMBIA

CALIFORNIA INSTITUTE OF TECHNOLOGY

UNIVERSITY OF CALIFORNIA

MONTANA STATE UNIVERSITY

UNIVERSITY OF NEVADA, RENO

NEW MEXICO STATE UNIVERSITY

OREGON STATE UNIVERSITY
UNIVERSITY OF OREGON

UNIVERSITY OF SOUTHERN CALIFORNIA

STANFORD UNIVERSITY

UNIVERSITY OF HAWAII

UNIVERSITY OF TOKYO

UNIVERSITY OF UTAH

WASHINGTON STATE UNIVERSITY

UNIVERSITY OF WASHINGTON 


\section{Pacific Journal of Mathematics}

\section{Vol. 97, No. $1 \quad$ January, 1981}

Charles A. Asmuth and Joe Repka, Tensor products for $S L_{2}(\mathfrak{k})$. II.

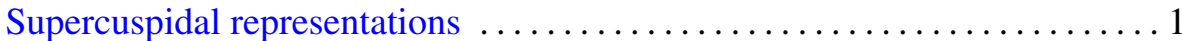

Joseph Barback, On finite sums of regressive isols . ................. 19

Matthew G. Brin and Daniel Russell McMillan, Jr., Generalized

three-manifolds with zero-dimensional nonmanifold set ............29

Kun Soo Chang, Converse measurability theorems for Yeh-Wiener space . . . 59

Christopher Brian Croke, A "maximal torus" type theorem for complete

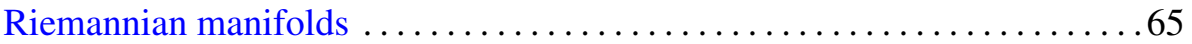

Gustave Adam Efroymson, Sums of squares in planar Nash rings . . . . . . 75

John Robert Fisher, Axiomatic radical and semisimple classes of rings . . . .81

Betty Kvarda, Consecutive integers for which $n^{2}+1$ is composite .......93

Roosevelt Gentry, New diagram proofs of the Hausdorff-Young theorem

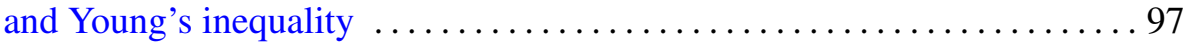

Patrick M. Gilmer, Topological proof of the $G$-signature theorem for $G$

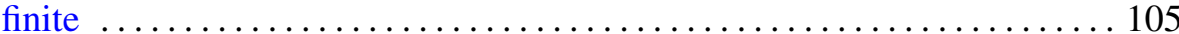

Chung Wei Ha, A noncompact minimax theorem .................. 115

James J. Hebda, Manifolds admitting taut hyperspheres ................ 119

Takayuki Kawada, Sample functions of Pólya processes ............. 125

Peter K. F. Kuhfittig, Common fixed points of nonexpansive mappings by

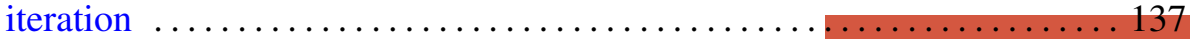

James Thomas Loats and Judith Roitman, Almost rigid Hopfian and dual

Hopfian atomic Boolean algebras .......................... 141

Roger McCann, On embedding semiflows into a radial flow on $l_{2} \ldots \ldots \ldots 151$

John McDonald, Closed orbits of convex sets of operators on the disk

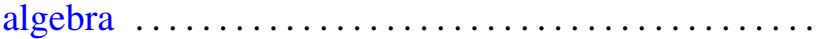

Mark D. Meyerson, Convexity and the table theorem .............. 167

Arnold William Miller, Generic Souslin sets . . . . . . . . . . . . . . . . 171

Takemi Mizokami, On the closed images of paracomplexes $\ldots \ldots \ldots \ldots \ldots 3$

Jagannadham Venkata Pakala and Thomas Stephen Shores, On

compactly packed rings $\ldots \ldots \ldots \ldots \ldots \ldots \ldots \ldots \ldots$

Andrew Pletch, Strong completeness in profinite groups

Wilbur Carrington Whitten, Inverting double knots

James Juei-Chin Yeh, Existence of strong solutions for stochastic

differential equations in the plane 